\title{
A PREDICTION METHOD FOR LOAD DISTRIBUTION IN THREADED CONNECTIONS
}

\author{
DONGMEI ZHANG \\ School of Mechanical and Electrical Engineering, North University of China, Taiyuan, China \\ e-mail: dongmei_zhang@163.com \\ Shiqiao Gao, Shaohua Niu, Haipeng Liu \\ State Key Laboratory of Explosion Science and Technology, Beijing Institute of Technology, Beijing, China
}

\begin{abstract}
A new method has been developed for predicting the load distribution along the thread portion of a bolt and nut connection. The calculated results were validated by comparison with three-dimensional finite element analysis and Yamamoto's method. It was shown that the load distribution predicted by the model in this paper was in good agreement with the results from finite element model, and the load ratio on the first thread by the prediction model and finite element model was slightly larger than the results from Yamamoto's method. In addition, the results of calculation and finite element analysis indicated that the decreasing of the lead angle could improve the load distribution, the increasing of the length of thread engaged could significantly improve the load bearing capacity of the first thread, and the adopting of a material with low stiffness for the nut with respect to the bolt could improve the load distribution slightly.
\end{abstract}

Keywords: load distribution, threaded connections, finite element analysis

\section{Introduction}

The bolted joint is a typical connection that is widely used for construction of structures from components. In order to ensure functionality of the joint, the load distribution in threaded connections is of concern.

The load distribution in threaded connections has been studied since the beginning of the century, but only a few of the most essential papers are referenced here (Goodier, 1940; Hetenyi, 1943; Motosh, 1975; Kenny and Patterson, 1985; Brutti and D'Eramo, 1987; Patterson and Kenny, 1986). The Sopwith (1948) theory for predicting the load distribution of thread fasteners is the most well known analytical model. The action of a number of strains is formulated by the axial extension of the bolt and compression of the nut. These strains include bending deflection of the thread, axial recession due to radial compression of the threads, and axial recession due to axial contraction of the bolt and expansion of the nut caused by radial pressure of the joints. Alternatively, Yamamoto (1980) proposed a procedure for calculating the deflection due to bending moment, shear loading and radial contraction and expansion on the bolt and the nut.

Due to the progress of the modern finite element method, the solution of contact problem becomes possible by using finite element software. Grosse and Mitchell (1990), Wileman et al. (1991), Lehnhoff and Wistehuff (1996), Chaaban and Muzzo (1991) and Chaaban and Jutras (1992) studied stresses in threaded connections by axisymmetrical finite element models by ASME Code. In order to investigate the helical effect on the thread connection, Chen and Shih (1999) built a three-dimensional bolt-nut assembly by ABAQUS. But there was a small hole on 
the center of the bolt, which slightly reduced the area of the applied load. Moreover, Eraslan and Inan (2010) built 3D finite element models of screws by Solidworks.

In order to achieve a more convenient prediction for the load distribution in screw threads, we developed a new analytical model to calculate the load distribution in the thread connection. Comparing the previous methods, especially Yamamoto's method which includes five deflections, there are only two main thread deflections in our method. In addition, we fully consider the ununiformity of load distribution on thread and use unit deflection per unit width under unit force. Meanwhile, a three-dimensional finite element model of the bolt-nut assembly is built to validate the prediction method.

\section{The new analytical model}

According to Sopwith's theory, the ISO triangle thread can be simplified into a cantilever beam with a variable cross section. The strain in the thickness direction is considered to be zero, namely, it is a plane strain problem. A coordinate system for the thread under axial concentrated load is established. The length direction of the thread is the $y$-axis, the depth direction is the $x$-axis, and the origin is located at the root of the thread, as shown in Fig. 1a. The original cross section of the thread is a triangle. The semi-angle of the thread is $\alpha$, the thread length is $2 a$, and the pitch diameter is $D$, as shown in Fig. 1b.

(a)

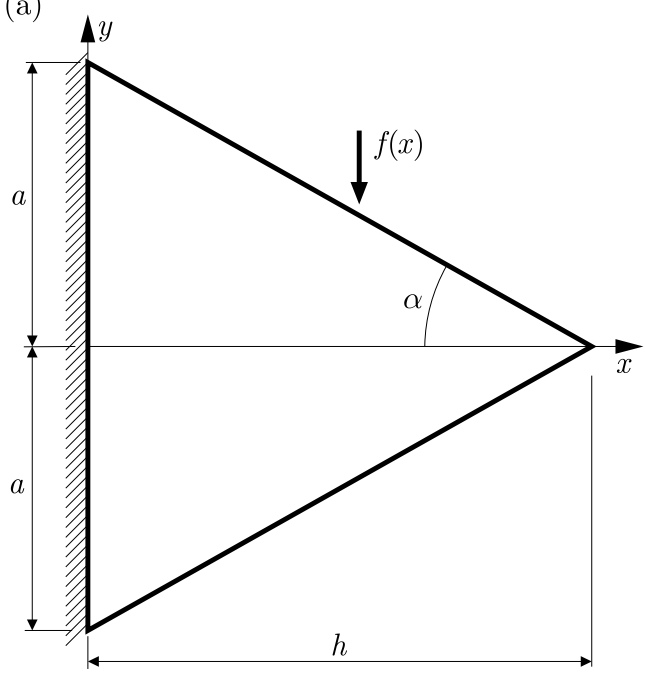

(b)

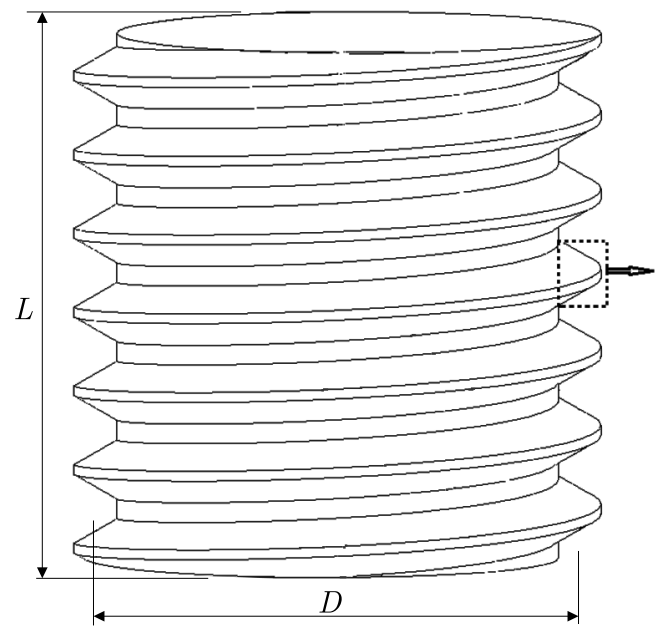

Fig. 1. (a) Thread structure and coordinate system, (b) bolt structure

The geometric equation of the upper half of the thread is

$y=a-k x$

where $k=\tan \alpha$ and $\alpha=30^{\circ}$.

The geometric equation of the lower half of the thread is

$$
y=k x-a
$$

The moment of inertia of one thread is

$$
I(x)=\iint_{S(x)} y^{2}(x) d S=\frac{2 \pi D}{3}(a-k x)^{3}
$$


where $a=k h, h$ is depth of the thread (as shown in Fig. 1a), therefore

$$
I(x)=\iint_{S(x)} y^{2}(x) d S=\frac{2 \pi D}{3} k^{3}(h-x)^{3}
$$

\subsection{The elastic deflection of thread}

According to Sopwith's theory, the thread under axial loading will deform due to several reasons. Owing to the above assumption, these deflections mainly include bending and shearing deflection of the thread. An ISO triangle thread can be seen as a cantilever beam under a concentrated load $P$ at $x=l_{1}$, which is shown in Fig. 2. The bending deflection $\delta_{1}$ and shearing deflection $\delta_{2}$ along the axial direction at $x=l_{1}$ can be obtained by the following functions.

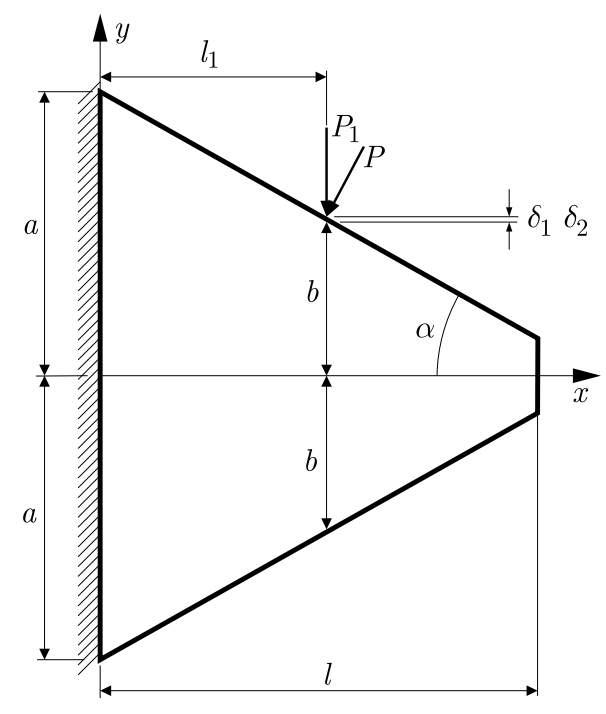

Fig. 2. Bending deflection and shearing deflection

\subsubsection{The deflection due to bending moment}

For a thread shown in Fig. 2, according to the theory of mechanics of materials, the static bending equation is

$$
E I(x) \frac{\partial^{2} w}{\partial x^{2}}=P_{1}\left(l_{1}-x\right) \quad x<l_{1}
$$

where $P_{1}$ is the axial force on one thread, $I(x)$ is the moment of inertia, $l_{1}$ is the distance from the action point of the force to the root of the thread, $E$ is Young's modulus and $w$ is the bending deflection. Substituting Eq. (2.4) into (2.5) leads to

$$
\frac{\partial^{2} w}{\partial x^{2}}=\frac{3 P_{1}}{2 \pi D E k^{3}} \frac{l_{1}-x}{(x-l)^{3}} \quad x<l_{1}
$$

where $l$ is height of the thread as shown in Fig. 2.

Primary integration of Eq. (2.6) is conducted in accordance with the assumption that the deflection angle at the root of the thread is zero, i.e., the boundary condition $\partial w / \partial x=0$ at $x=0$ and the deflection angle can be expressed as

$$
\frac{\partial w}{\partial x}=\frac{-3 P_{1}}{2 \pi D E k^{3}}\left(\frac{1}{x-l}+\frac{l-l_{1}}{2(x-l)^{2}}+\frac{1}{l}-\frac{l-l_{1}}{2 l^{2}}\right) \quad x<l_{1}
$$


Primary integration of Eq. (2.7) is conducted on the assumption that the displacement at the root of the thread is zero, i.e., the boundary condition $w=0$ at $x=0$ is

$$
w=\frac{-3 P_{1}}{2 \pi D E k^{3}}\left[\ln \frac{l-x}{l}-\frac{l-l_{1}}{2(x-l)}+\left(\frac{1}{l}-\frac{l-l_{1}}{2 l^{2}}\right) x-\frac{l-l_{1}}{2 l}\right] \quad x<l_{1}
$$

Under the unit force, the bending deflection of one thread at $x=l_{1}$ is

$$
\delta_{1}=\frac{-3}{2 \pi D E k^{3}}\left[\ln \frac{l-l_{1}}{l}+\frac{1}{2}+\left(\frac{1}{l}-\frac{l-l_{1}}{2 l^{2}}\right) l_{1}-\frac{l-l_{1}}{2 l}\right]
$$

Because the deformation of thread is treated as a plane deformation, under the unit force, the unit deflection due to bending moment can be expressed as

$$
\delta_{1}=\frac{-3\left(1-\nu^{2}\right)}{2 \pi D E k^{3}}\left[\ln \frac{l-l_{1}}{l}+\frac{1}{2}+\left(\frac{1}{l}-\frac{l-l_{1}}{2 l^{2}}\right) l_{1}-\frac{l-l_{1}}{2 l}\right]
$$

\subsubsection{The deflection due to shear loading}

At $x=l_{1}$, the shear stress for the thread can be expressed as

$$
\tau(x)=\frac{P_{1}}{\pi D} \frac{1}{2(a-k x)}
$$

Therefore, the shearing deflection of one thread should be

$$
\Delta(x)=\frac{\tau(x)}{G} L
$$

where the $L$ is total length of the thread as shown in Fig. $1 b$.

The shearing deflection of the unit length $(L \equiv 1)$ is

$$
\delta_{U}(x)=\frac{\tau(x)}{G}
$$

where $G$ is shear modulus, and $G=E[2(1+\nu)]$. Therefore, the unit shearing deflection of one thread under the unit force $\left(P_{1} \equiv 1\right)$ can be expressed as

$$
\delta(x)=\frac{1+\nu}{E \pi D} \frac{1}{a-k x}
$$

According to the boundary condition $\delta(x)=0$ for $x=0$ and $\delta(x)=\delta_{2}$ for $x=l_{1}$, integration of Eq. (2.14) leads to

$$
\delta_{2}=\frac{1+\nu}{E \pi D} \int_{0}^{l_{1}} \frac{d x}{a-k x}=\frac{1+\nu}{k E \pi D} \ln \frac{a}{b}
$$

According to Yamamoto (1980), the load $P$ is located at the center of the original triangle of the thread, namely

$$
l_{1}=\frac{1}{2} l \quad b=\frac{1}{2} a
$$

Therefore, for the bolt and nut under the unit force, substituting of Eqs. (2.16) into Eqs. (2.10) and (2.15), respectively, the elastic deformation of unit length thread in the direction of the helix is

$$
\begin{aligned}
& \delta_{b}=\delta_{1 b}+\delta_{2 b}=\frac{0.5\left(1-\nu_{b}^{2}\right)+1.2\left(1+\nu_{b}\right)}{\pi D E_{b}} \\
& \delta_{n}=\delta_{1 n}+\delta_{2 n}=\frac{0.5\left(1-\nu_{n}^{2}\right)+1.2\left(1+\nu_{n}\right)}{\pi D E_{n}}
\end{aligned}
$$


where $\delta_{1 b}$ and $\delta_{2 b}$ represent the bending and shearing deflection of bolt, respectively, $\delta_{1 n}$ and $\delta_{2 n}$ represent the bending and shearing deflection of the nut. For the ISO triangle thread, there is $k=\tan 30^{\circ}$. For the threaded connection structure composed of the bolt and nut, the total elastic deformation of the unit length thread in the direction of the helix is

$$
\delta_{y}=\delta_{b}+\delta_{n}
$$

where $\delta_{b}$ and $\delta_{n}$ represent the total deflection of the bolt and nut, respectively.

\subsection{Thread stiffness}

For the thread on the bolt, the stiffness of the unit length thread under the unit force in the direction of the helix is

$$
k_{b u}=\frac{1}{\delta_{b}}
$$

Because the total length of the thread is $\pi D$, the total axial stiffness of the thread on the bolt under the unit force is

$$
K_{b}=\pi D k_{b u}=\frac{\pi D}{\delta_{b}}
$$

Similarly, for the thread on the nut, the stiffness of the unit length under the unit force in the direction of the helix is

$$
k_{n u}=\frac{1}{\delta_{n}}
$$

The thread stiffness of the thread on the nut under the unit force is

$$
K_{n}=\pi D k_{n u}=\frac{\pi D}{\delta_{n}}
$$

If one thread is considered to be a spring, the threaded connection that consists of several threads is a parallel spring. Thus, for the bolt, the total stiffness of $n$ thread is $n K_{b}$. If the pitch is $p$, then the stiffness of the unit axial length under the unit force is regarded as

$$
k_{b y}=\frac{K_{b}}{p}=\frac{\pi D k_{b u}}{p}
$$

If the lead angle of the helix is $\beta$, then $\tan \beta=p /(\pi D)$. For the bolt and nut, the stiffness of the unit axial length under the unit force is respectively

$$
k_{b y}=\frac{k_{b u}}{\sin \beta}=\frac{1}{\delta_{b}} \sin \beta \quad k_{n y}=\frac{k_{n u}}{\sin \beta}=\frac{1}{\delta_{n}} \sin \beta
$$

For the threaded connection structure composed of the bolt and nut, the total stiffness of the unit axial length is

$$
k_{y}=\frac{1}{\frac{1}{k_{b y}}+\frac{1}{k_{n y}}}=\frac{1}{\left(\delta_{b}+\delta_{n}\right) \sin \beta}=\frac{1}{\delta_{y} \sin \beta}
$$

Because the stiffness is the ratio of the force to deflection, the axial deflection of the thread body is

$$
\Delta_{y}=\frac{1}{k_{y}} \frac{\partial P}{\partial y}
$$

where $\partial P / \partial y$ is the axial force on the unit length thread. The preceding equation indicates that the axial deflection of the thread body varies directly with load distribution. 


\subsection{Axial load distribution in threaded connection}

Figure 3 shows that the threaded connection structure includes a fixed nut body and a bolt body. In the bolt body under a compressing force $f_{B}$, the force will be transferred to the nut body through the threads. The structure is elastic. Thus, the bolt body, the nut body or the thread body will exhibit deflection under the force. However, the forms of forces on the bolt body and the nut body differ from those on the thread body. Furthermore, their load distributions also vary. Thus, the deformation modes of the bolt body and the nut body differ from those of the thread body. However, the bolt body and the nut body are joined by the thread body. Thus, their deformations are compatible.

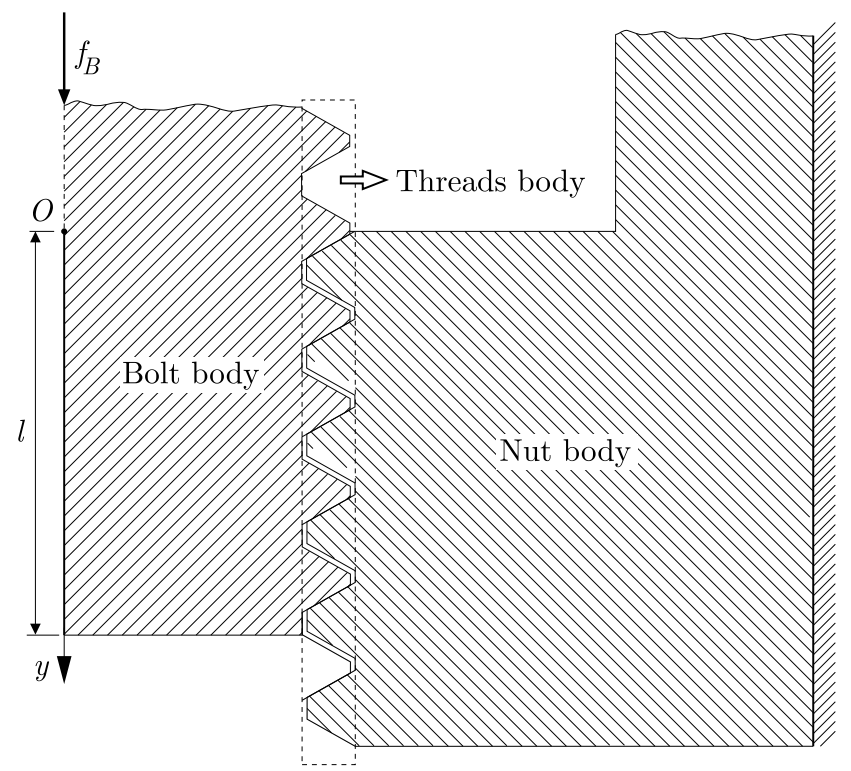

Fig. 3. Threaded connection structure

The region of the compressing force on the thread body ranges from 0 to $l$, where $l$ is the engaged length of the bolted joint. Thus, the strain $\varepsilon_{b}$ generated by the bolt body under the compressing force $f(y)$ at the location $y$ is

$$
\varepsilon_{b}=\frac{f(y)}{S_{b} E_{b}}
$$

where $S_{b}$ is the cross-sectional area of the bolt body.

Similarly, strain $\varepsilon_{n}$ generated by the bolt body under the compressing force $f(y)$ is

$$
\varepsilon_{n}=\frac{f(y)}{S_{n} E_{n}}
$$

where $S_{n}$ is the cross-sectional area of the nut body.

For the thread body, the load $f$ is distributed along the direction of the helix and along the axial direction $y$. The thread deformation analysis indicates that the deflection and displacement of threads on the bolt body is related to the gradient of force distribution, i.e.,

$$
\delta_{b}=\frac{1}{k_{b y}} \frac{\partial f}{\partial y}
$$

Similarly, the deflection of the thread body on the nut body is

$$
\delta_{n}=\frac{1}{k_{n y}} \frac{\partial f}{\partial y}
$$


Their displacement gradients are respectively

$$
\frac{\partial \delta_{b}}{\partial y}=\frac{1}{k_{b y}} \frac{\partial^{2} f}{\partial y^{2}} \quad \frac{\partial \delta_{n}}{\partial y}=\frac{1}{k_{n y}} \frac{\partial^{2} f}{\partial y^{2}}
$$

In order to make the deformation compatible, $\varepsilon_{b}, \varepsilon_{n}, \delta_{b}$ and $\delta_{n}$ must meet the following relationship

$$
\varepsilon_{b}+\varepsilon_{n}=\frac{\partial \delta_{b}}{\partial y}+\frac{\partial \delta_{n}}{\partial y}
$$

Substitution of Eqs. (2.27), (2.28) and (2.31) into Eq. (2.32) leads to

$$
\lambda^{2} f=\frac{\partial^{2} f}{\partial y^{2}}
$$

where

$$
\lambda=\sqrt{\frac{\frac{1}{S_{b} E_{b}}+\frac{1}{S_{n} E_{n}}}{\frac{1}{k_{n y}}+\frac{1}{k_{b y}}}}
$$

The solution to Eq. (2.32) is

$$
f=C_{1} \sinh \lambda y+C_{2} \cosh \lambda y
$$

By the boundary conditions $f(y)=f_{B}$ for $y=0$ and $f(y)=0$ for $y=l$, we can obtain

$$
C_{2}=f_{B} \quad C_{1}=-\frac{\cosh \lambda l}{\sinh \lambda l} f_{B}
$$

Substituting $C_{1}$ and $C_{2}$ into Eq. (2.34) leads to

$$
f(y)=f_{B}\left(-\frac{\cosh \lambda l}{\sinh \lambda l} \sinh \lambda y+\cosh \lambda y\right)
$$

where $f_{B}$ is the load on the first thread of the bolt. The load along the axial direction $y$ can be calculated by Eq. (2.35). This distribution is more precise than Yamamoto's method because we considered the load distribution along the helix of the screw.

\section{Finite element model}

A group of ISO metric screw threads M36 are used to study the load distribution and the effect of pitch, material and length of the thread engagement. The parameters of all screws are listed in Table 1. Commercial finite element software ANSYS is used for modeling and analysis. The model geometry is meshed by 8 node hexahedron elements (SOLID185). The contact and target elements are TARGE169 and CONTA172, respectively. The friction coefficient changes between 0.150 .2 according to the material of the thread. Figure 4 shows 3-D finite element models of the standard bolt assembly and the stress distribution on the screw under the external load. There is no sliding between the bolt and nut threads because friction coefficients are large enough to prevent sliding. In the models, the axial loading is applied to the top surface of the bolt, and the outer bottom surface of nut is assumed fixed. Convergence study is carried out on the initial finite element model by decreasing the element size near the threads. The smallest element size is $0.25 \mathrm{~mm}$ by $0.25 \mathrm{~mm}$ and the total number of element is about 80000 . There is no significant improvement in the accuracy by using smallest elements. 
Table 1. Parameters of the thread

\begin{tabular}{|c|c|c|c|c|c|}
\hline Speci- & Nominal diameter & Pitch & Engaged length & \multicolumn{2}{|c|}{ Materials } \\
\hline No. & $d[\mathrm{~mm}]$ & {$[\mathrm{mm}]$} & $l[\mathrm{~mm}]$ & bolt & nut \\
\hline 1 & 36 & 4 & 28 & \multirow{8}{*}{ steel } & \multirow{8}{*}{ steel } \\
\hline 2 & 36 & 3 & 28 & & \\
\hline 3 & 36 & 2 & 28 & & \\
\hline 4 & 36 & 1.5 & 28 & & \\
\hline 5 & 36 & 4 & 24 & & \\
\hline 6 & 36 & 4 & 20 & & \\
\hline 7 & 36 & 4 & 16 & & \\
\hline 8 & 36 & 4 & 12 & & \\
\hline 9 & 36 & 4 & 28 & steel & copper \\
\hline 10 & 36 & 4 & 28 & steel & brass \\
\hline 11 & 36 & 4 & 28 & steel & aluminum \\
\hline 12 & 36 & 4 & 28 & aluminum & steel \\
\hline
\end{tabular}

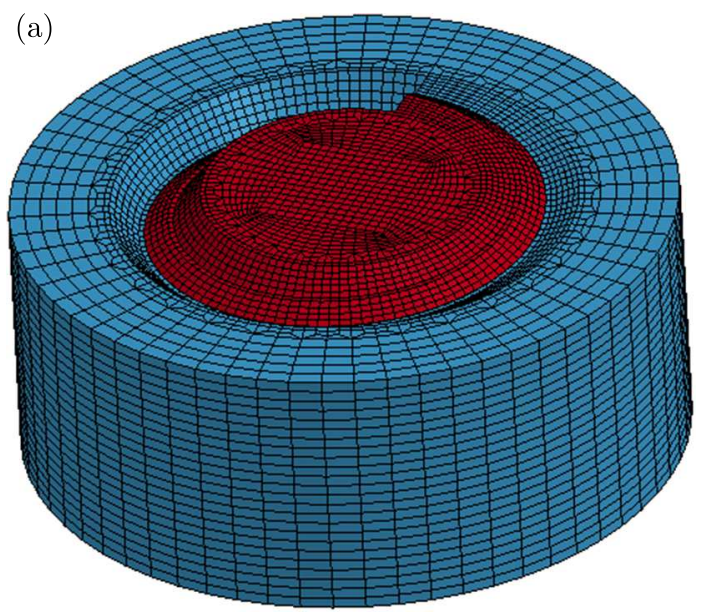

(b)

(c)

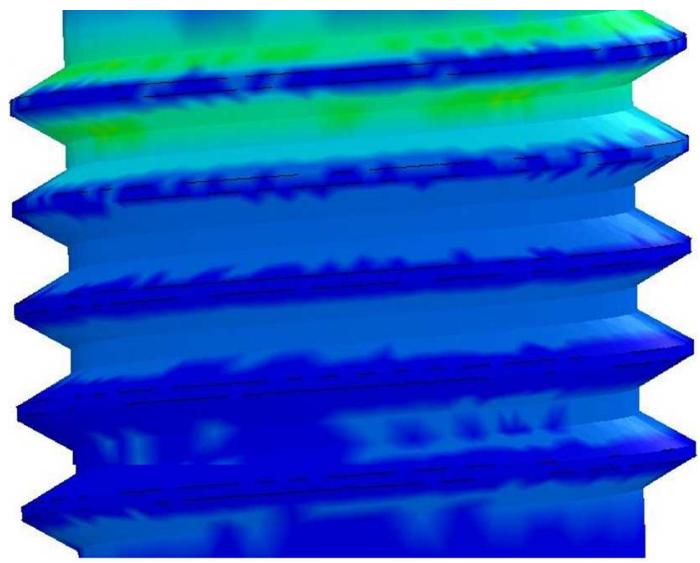

Fig. 4. 3-D model mesh of standard threaded connection: (a) bolted joint assembly, (b) bolt, (c) contour plot of the screw stress 
Table 2. Parameters of material

\begin{tabular}{|c|c|c|c|}
\hline Material & $\begin{array}{c}\text { Young's modulus } \\
E[\mathrm{GPa}]\end{array}$ & $\begin{array}{c}\text { Poisson's ratio } \\
\nu[-]\end{array}$ & $\begin{array}{c}\text { Density } \\
\rho\left[\mathrm{kg} / \mathrm{m}^{3}\right]\end{array}$ \\
\hline \hline Steel & 211 & 0.269 & 7890 \\
\hline Copper & 137 & 0.310 & 8980 \\
\hline Brass & 105 & 0.320 & 8500 \\
\hline Aluminum & 69 & 0.330 & 2700 \\
\hline
\end{tabular}

An elastic material is used throughout this work. A uniform pressure loading $p$ is applied to the top root surface of the bolt. So, the total force due to the applied pressure can be expressed as

$$
f_{B}=p \frac{\pi d^{2}}{4}
$$

where $d$ is the nominal diameter of the bolt.

\section{Result and discussion}

For thread No. 1, the result of the load distribution on each thread by Yamamoto's method, finite element model and the prediction model developed in this paper can be shown in Fig. 5 .

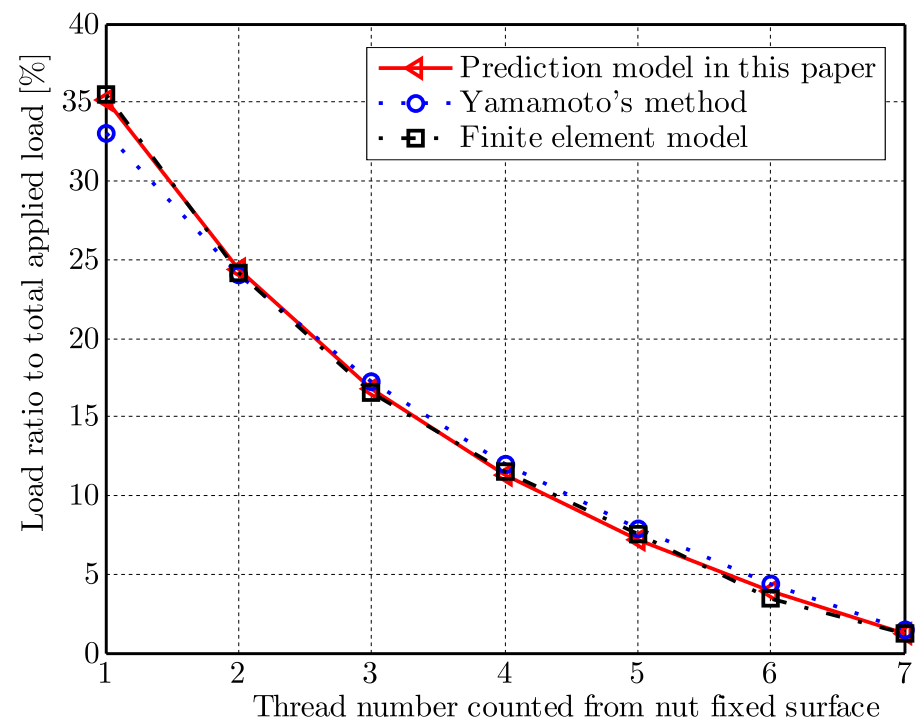

Fig. 5. The load ratio on each thread for bolted joints No. 1

Figure 5 shows that the load distribution obtained from the model in this paper are in a close agreement with the finite element analysis. It is a load corresponding to the pitch section of the thread. The load distribution with prediction from the analytical model and finite element model is shown to be slightly larger than the results of Yamamoto's method. For the FEM calculation, the axial component of stress is utilized for the load on thread and the load ratio is the ratio of the load on thread to the load on the surface of the bolt. The load on thread can be calculated by equation (2.32) for the prediction model, and it is the average value of some element (all the elements along the thread edge) for the FEM calculation. From the figure, we can see that the loading ratio is quite similar on each thread in the case of the analytical model and finite element model for all specimens. 
There are four pitches which have been used in this Section, namely $4 \mathrm{~mm}, 3 \mathrm{~mm}, 2 \mathrm{~mm}$, $1.5 \mathrm{~mm}$, and the corresponding lead angles are $2.1804^{\circ}, 1.6068^{\circ}, 1.0511^{\circ}, 0.7810^{\circ}$, respectively. The load ratio of the prediction model in this paper with different pitch in Fig. 6 shows that the decreasing of the pitch can improve the load distribution and reduces the load bearing on the first thread.

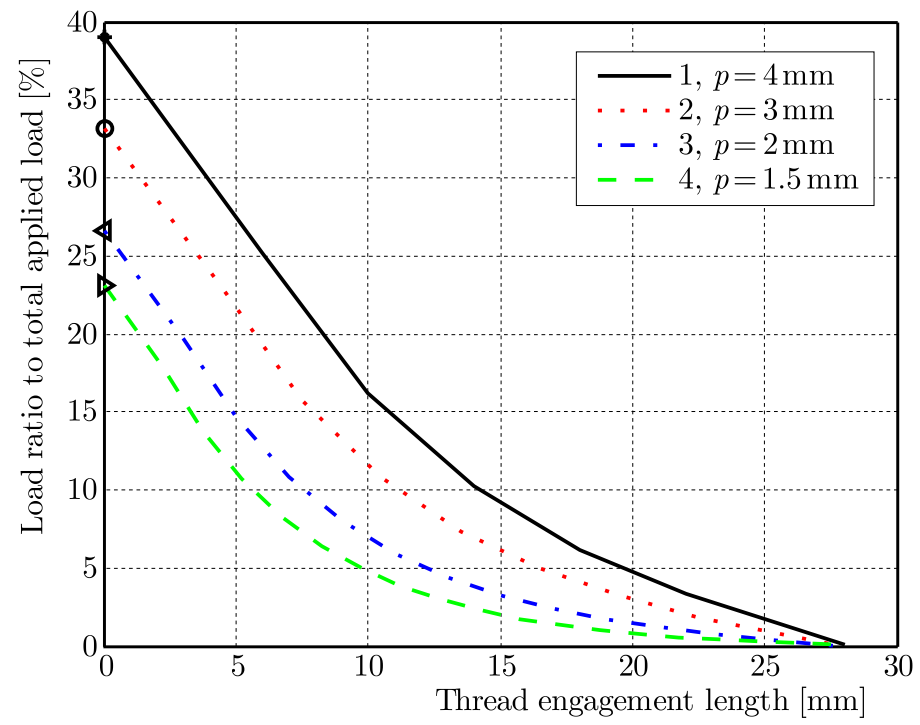

Fig. 6. The load ratio on each thread for bolted joints No. 1, 2, 3, and 4

Figure 7 is the load ratio of the prediction model and finite element model with length of thread engagement $28 \mathrm{~mm}, 24 \mathrm{~mm}, 20 \mathrm{~mm}, 16 \mathrm{~mm}, 12 \mathrm{~mm}$, respectively. The figures show that the load ratio is modified greatly with the increasing of thread engagement length. And the longer the length of thread engagement, the smaller the load bearing on the first thread. But when the length is more than $20 \mathrm{~mm}$, the effect is very slight.

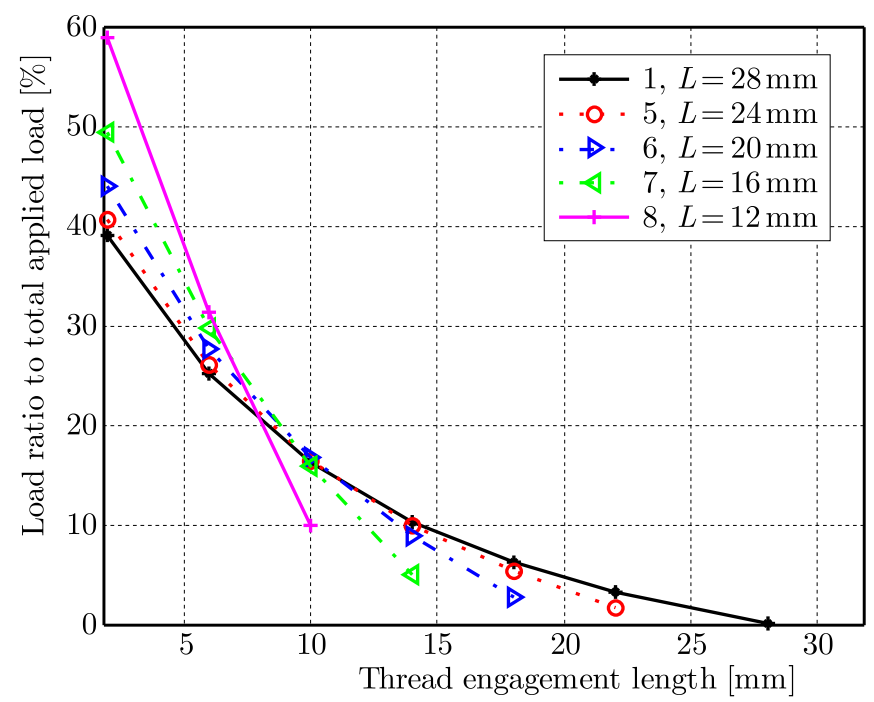

Fig. 7. The load ratio on each thread for bolted joints No. 1, 5, 6, 7 and 8

In addition, when Young's modulus the of bolt $E_{b}$ is larger than that of the nut $E_{n}$, the load distribution is improved slightly. The calculation result by the prediction model for specimens No. 1, 9, 10, 11, 12 whose bolts and nuts are made of different materials, are shown in Fig. 8. 


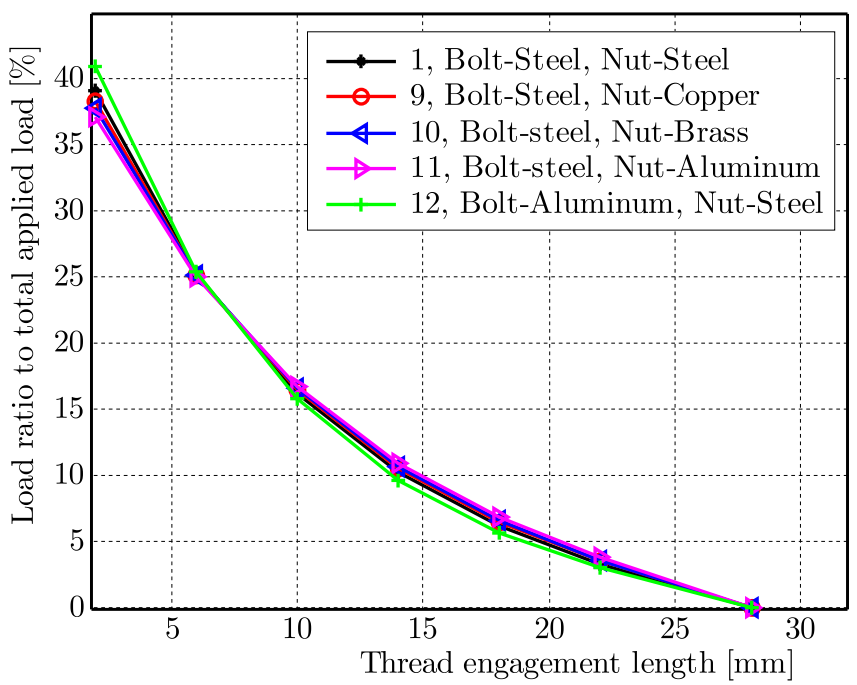

Fig. 8. The load ratio on each thread for bolted joints No. 1, 9, 10, 11 and 13

\section{Conclusions}

The load distributions on the thread connection by the analytical model and finite element model have been studied. The results can be concluded as follows.

1. The load distributions obtained from the model in this paper are in good agreement with finite element analysis and Yamamoto's method.

2. The results of the analytical model in this paper are nearer to the finite element analysis than Yamamoto's method, because we considered the load distribution along the helix of the screw.

3. The effect of pitch on the load distribution of the threaded assembly is very obvious. A decrease in the pitch not only can improve the load distribution, but also can reduce the load ratio in the first thread.

4. The load distribution of the first thread is significantly decreased by a properly increased length of the thread engagement.

5. Adopting a material with low stiffness for the nut with respect to the bolt can improve the load distribution slightly.

\section{References}

1. Brutti C., D'Eramo M., 1987, Methods to evaluate load distribution in screw threads, Proceedings of 2th Canadian Conference of Applied Mechanics, Edmonton, 74-75

2. ChaAban A., Jutras M., 1992, Static analysis of buttress threads using the finite element method, Journal of Pressure Vessel Technology, 114, 209-212

3. Chatban A., Muzzo U., 1991, Finite element analysis of residual stresses in threaded end closure, Journal of Pressure Vessel Technology, 113, 398-401

4. Chen J.J., Shin Y.S., 1999, A study of the helical effect on the thread connection by three dimensional finite element analysis, Nuclear Engineering and Design, 191, 2, 109-116

5. Eraslan O., Inan O., 2010, The effect of design on stress distribution in a solid screw implant: a 3D finite element analysis, Clinical Oral Investigations, 14, 411-416

6. Goodier J.N., 1940, The distribution of load on the threads of screws, Transactions ASME, 62, $10-16$ 
7. Grosse I.R., Mitchell L.D., 1990, Non-linear axial stiffness characteristic of axisymmetric bolted joint, Journal of Mechanical Design, 112, 442-449

8. Hetenyi M., 1943, A photoelastic study of bolt and nut fastenings, Transactions ASME, 65, 93-100

9. Kenny B., Patterson E.A., 1985, Load and stress distribution in screw threads, Experimental Mechanics, 25, 208-213

10. Lehnhoff T.F., Wistehuff W.E., 1996, Non-linear effects on the stress and deformations of bolted joints, Journal of Mechanical Design, 118, 54-58

11. Motosh N., 1975, Load distribution on threads of titanium tension nut and steel bolts, Transactions ASME, 97, 162-166

12. Patterson E.A., Kenny B., 1986, A modification to the theory for the load distribution in conventional nuts and bolts, Proceedings of the Institution of Mechanical Engineers, 21, 1, 17-23

13. Sopwith D.G., 1948, The distribution of load in screw threads, Proceedings of the Institution of Mechanical Engineers, 159, 373-383

14. Wileman J., Choudhury M., Green I., 1991, Computation of member stiffness in bolted connections, Journal of Mechanical Design, 113, 432-437

15. Yамамото A., 1980, The Theory and Computation of Threads Connection (in Japanese), Yokendo, Tokyo

Manuscript received June 12, 2016; accepted for print August 7, 2017 